\title{
Pulse Rate Variability Analysis to Enhance Oximetry as at-Home Alternative for Sleep Apnea Diagnosing
}

\author{
Gonzalo C. Gutiérrez-Tobal, Daniel Álvarez, Fernando Vaquerizo-Villar, \\ Verónica Barroso-García, Adrián Martín-Montero, Andrea Crespo, \\ Félix del Campo, and Roberto Hornero
}

\begin{abstract}
This study focuses on the at-home Sleep apnea-hypopnea syndrome (SAHS) severity estimation. Three percent oxygen desaturation index $\left(O D I_{3}\right)$ from nocturnal pulse-oximetry has been commonly evaluated as simplified alternative to polysomnography (PSG), the standard in-hospital diagnostic test. However, $\mathrm{ODI}_{3}$ has shown limited ability to detect SAHS as it only sums up information from desaturation events. Other physiological signs of SAHS can be found in respiratory and cardiac signals, providing additional helpful data to establish SAHS and its severity. Pulse rate variability time series (PRV), also derived from nocturnal oximetry, is considered a surrogate for heart rate variability, which provides both cardiac and respiratory information. In this study, 200 oximetric recordings obtained at patients home were involved, divided into training (50\%) and test (50\%) groups. $\mathrm{ODI}_{3}$ and PRV were obtained from them, the latter being characterized by the extraction of statistical features in time domain, as well as the spectral entropy from the commonly used very low $(0-0.04 \mathrm{~Hz}$.), low $(0.04-0.15 \mathrm{~Hz}$.$) , and high (0.15-0.4 \mathrm{~Hz}$.) frequency bands. The $\mathrm{ODI}_{3}$ and PRV features were joined in a multi-layer perceptron artificial neural network (MLP), trained to estimate the apnea-hypopnea index (AHI), which is the PSG-derived parameter used to diagnose SAHS. Our results showed that single $\mathrm{ODI}_{3}$ rightly assigned $62.0 \%$ of the subjects from the test group into one out the four SAHS severity degrees, reaching 0.470 Cohens kappa, and 0.840 intra-class correlation
\end{abstract}

G. C. Gutiérrez-Tobal $(\bowtie)$ - D. Álvarez · F. Vaquerizo-Villar V. Barroso-García · A. Martín-Montero - A. Crespo · F. del Campo $\cdot$ R. Hornero

Biomedical Engineering Group, Universidad de Valladolid,

Valladolid, Spain

e-mail: gonzalo.gutierrez@gib.tel.uva.es; gguttob@gmail.com URL: http://www.gib.tel.uva.es/

D. Álvarez · A. Crespo · F. del Campo

Sleep Unit, Hospital Universitario Rio Hortega, Valladolid, Spain coefficient (ICC) with the actual AHI (accuracies of 90.0, 88.0 and $82.0 \%$ in the increasing AHI cutoffs used to define SAHS severity). By contrast, our MLP model rightly assigned $75.0 \%$ of the subjects into their corresponding SAHS severity level, reaching $0.614 \kappa$ and 0.904 ICC (accuracies of 93.0, 88.0 and 90.0\%). These results suggest that SAHS diagnosis could be accurately conducted at-patients home by combining $\mathrm{ODI}_{3}$ and PRV from nocturnal oximetry

\section{Keywords}

Sleep apnea $・$ Pulse rate variability - Oximetry

Artificial neural network $\bullet$ Home diagnosis

\section{Introduction}

The sleep apnea-hypopnea syndrome (SAHS) is a chronic and prevalent disease in which patients show recurrent episodes of respiratory pauses (apneas) and airflow reductions (hypopneas) while sleeping [1]. Apneic events boost a number of undesirable physiological processes that lead to a harmful impact both in health and quality of life of affected people [2].

The number of apneas and hypopneas per hour of sleep, i.e., the apnea-hypopnea index (AHI), is the parameter used to determine the presence and severity of SAHS [1, 3]. Clinical specialists estimate AHI by examining multiple physiological signals recorded during in-lab nocturnal polysomnography (PSG), which is the standard diagnostic test [3]. However, simplifying SAHS diagnosis has become a major issue for biomedical engineering due to limitations related to the PSG complexity, costs, and demand of time from physicians [3]. These drawbacks, together with the high prevalence of SAHS [3], imply a limited availability of specialized facilities, which derives in long waiting lists and delays the access to both diagnosis and treatment [3]. 
In past years, a wide range of simpler diagnostic alternatives have been evaluated. In contrast to 32 signals recorded during PSG, most of the alternatives focused on analyzing a single one [4, 5]. Nocturnal pulse-oximetry (NPO) is a simple test often used for this purpose [6, 7]. It only requires a sensor on a finger to record the blood oxygen saturation $\left(\mathrm{SpO}_{2}\right)$. This signal monitors the oxygen in the hemoglobin of the blood, which falls due to apneic events [1].

Promising results have been derived from the investigation of the $\mathrm{SpO}_{2}$ signal, which include the use of automatic signal processing techniques and the assessment of clinically-used oxygen desaturation indexes (ODI) [6, 7]. However, recent studies have reported substantial redundancy between the $3 \%$ ODI $\left(O D I_{3}\right)$, and the remaining information usually extracted from $\mathrm{SpO}_{2}$ [8]. Moreover, SAHS is known to change the normal profile of the cardiac signals by the recurrence of bradycardia/tachycardia patterns [4]. Particularly, a recent work from our group reported that spectral entropy (SE) from VLF (0-0.04 Hz), LF (0.04$0.15 \mathrm{~Hz})$, and $\mathrm{HF}(0.15-0.40 \mathrm{~Hz})$ bands of the heart rate variability signal (HRV) provides more useful information than the corresponding power-based traditional features [9]. This kind of information is not available when using $\mathrm{SpO}_{2}$ signal alone. Hence, we hypothesize that the usefulness of $\mathrm{ODI}_{3}$ to simplify SAHS diagnosis test can be improved adding cardiac data of interest.

In this regard, NPO sensor is not only able to record $\mathrm{SpO}_{2}$ but pulse rate too. Several studies have evaluated the use of cardiac information obtained from the pulse rate variability signal (PRV) as a surrogate of HRV [10, 11]. These works showed the usefulness of PRV in pediatric SAHS context. However, they did not evaluate adult subjects, they focused on the analysis of the classic spectral power-based HRV parameters [10], or only used time-domain PRV information to detect specific apneic related events [11]. Additionally, the signals used in these works were acquired in a laboratory, i.e., under supervised conditions.

In accordance with the above mentioned, we aim at evaluating the joint usefulness of $\mathrm{ODI}_{3}$ from $\mathrm{SpO}_{2}$ together with time and frequency domain features obtained from PRV. The extraction of $S E$ from VLF, LF, and HF of PRV spectrum is proposed. Additionally, first to fourth order statistics $(M t 1-M t 4)$ are also obtained with the purpose of characterizing the whole PRV time series. Moreover, in contrast to previous studies, both PSG and NPO are conducted at patients home. Hence, our proposal can be evaluated under those conditions which best reflect the usual sleep environment and behavior of patients. The eight features extracted from NPO ( $\mathrm{SpO}_{2}$ and PRV) are subsequently used as the only source of information to train a multi-layer perceptron (MLP) artificial neural network with ability to automatically estimate AHI. We chose MLP in view of its success in previous studies focused on automatic AHI estimation $[12,13]$. The diagnostic performance of this estimated AHI is compared with $\mathrm{ODI}_{3}$ to assess whether the use of PRV information can improve the ability of oximetry to detect SAHS and its severity.

\section{Subjects and Signals}

The study involved 200 adult subjects (67.5\% males). All of them where referred to the sleep unit of the Hospital Universitario Rio Hortega in Valladolid (Spain) due to clinical suspicious of SAHS. The subjects gave their informed consent to participate in the study and the Ethics Committee of the Hospital approved the protocol. A physician examined at-home PSG (Embletta MPR ST+, Embla Systems, USA) tests from all patients to compute an AHI for each of them. Apneas and hypopneas were scored following the rules of the American Academy of Sleep Medicine (AASM) [1]. According to the computed AHI, subjects were diagnosed in four SAHS-severity degrees: 12 no-SAHS $(\mathrm{AHI}<5 \mathrm{e} / \mathrm{h}), 46$ mild $(5 \leq \mathrm{AHI}<15 \mathrm{e} / \mathrm{h}), 46$ moderate $(15 \leq \mathrm{AHI}<30 \mathrm{e} / \mathrm{h})$, and 96 severe $(\mathrm{AHI} \geq 30 \mathrm{e} / \mathrm{h})$. The sample was randomly divided into a training $(50 \%)$ and a test $(50 \%)$ set, with the two of them having the same number of subjects from each SAHS-severity degree. Table 1 shows the demographic and clinical data of the subjects under study for the whole group and the training and test sets.

A NPO (WristOx ${ }_{2}^{T M}$, Nonin, USA) was conducted at the same time that each PSG. $\mathrm{SpO}_{2}$ and PRV were obtained at sampling rates of $1 \mathrm{~Hz}$ and $3 \mathrm{~Hz}$, respectively. Artifacts were removed from both of them. In $\mathrm{SpO}_{2}$, zero values and differences between consecutive samples $\geq 4 \%$ were removed and substituted by interpolated data [14]. In PRV, values $<0.33 \mathrm{~s}$ or $>1.5 \mathrm{~s}$, as well as differences in consecutive PRV values $>0.66$, were considered arrhythmia-related artifacts [4]. All of them were also removed and substituted by interpolated samples [4].

Table 1 Demographic and clinical data of the subjects under study

\begin{tabular}{l|l|l|l}
\hline Data & All & Training & Test \\
\hline Subjects $(n)$ & 200 & 100 & 100 \\
\hline Male $(\%)$ & 67.5 & 64.0 & 71.0 \\
\hline Age $($ years $)$ & $55.4 \pm 12.6$ & $55.0 \pm 11.9$ & $55.8 \pm 13.3$ \\
\hline BMI $\left(\mathrm{Kg} / \mathrm{m}^{2}\right)$ & $29.5 \pm 5.3$ & $29.7 \pm 5.3$ & $29.3 \pm 5.4$ \\
\hline AHI $(\mathrm{e} / \mathrm{h})$ & $34.3 \pm 24.5$ & $35.1 \pm 25.3$ & $33.5 \pm 23.8$ \\
\hline
\end{tabular}




\section{Methodology}

\section{1 $\mathrm{SpO}_{2}$ and PRV Features}

One feature was obtained from the $\mathrm{SpO}_{2}$ signal $\left(\mathrm{ODI}_{3}\right)$ whereas 7 more were extracted from PRV: first- to fourth order statistical moments $M t 1-M t 4$ in time domain and the spectral entropy from the $\operatorname{VLF}\left(S E_{V L F}\right), \operatorname{LF}\left(S E_{L F}\right)$, and $\mathrm{HF}\left(S E_{H F}\right)$ frequency bands. Next we briefly describe each of them:

- $\mathrm{ODI}_{3}$ is a clinical parameter widely used to help in SAHS diagnosis. It computes the number of drops from the $\mathrm{SpO}_{2}$ signal baseline $\geq 3 \%$, divided by the number of hours of the recording [14].

- Mt1-Mt4 are the well-known mean (Mt1), standard deviation (Mt2), skewness (Mt3), and kurtosis (Mt4), which characterize the central tendency, dispersion, asymmetry, and peakedness of a time series, respectively. They have shown its utility to analyze oximetric signals in the past [12].

- $S E$ measures the flatness of the spectrum of a biomedical signal [9]. Higher $S E$ values (closer to 1) are reached when the spectral power is spreaded throughout frequencies. By contrast, $S E$ values closer to 0 are reached in the presence of spectrums where power is condensed [9]. $S E$ can be computed by applying Shannon's entropy to a normalized version of the power spectral density in a frequency range [9].

These eight features were used to characterize the recordings of each subject under study and train (and test) a MLP model with ability to automatically estimate AHI.

\subsection{Multi-layer Perceptron Artificial Neural Network}

MLP is a supervised learning algorithm typically arranged in three fully connected layers (input, hidden, and output) [13]. The layers are formed by neurons, each of them characterized by an activation function $g()$ and their connections (or weights) to neurons from other layers $\left(w_{i, j}\right.$, being $i$ and $j$ different layers). In this study, the input layer has eight neurons due to the number of the extracted features. Moreover, according to the AHI regression task, a single unit with a linear activation function was used in the output layer. Linear activation functions were also used for each neuron in the hidden layer $\left(N_{H}\right)$. The number of hidden neurons finally arranged is a tuning parameter optimized using the training set [13]. In order to prevent overfitting, a regularization parameter $(\alpha)$ was introduced during the MLP training process [13]. It was also optimized along with $N_{H}$. All weights $w_{i, j}$ were optimized using the sum of squares error function minimization criterion by means of the scaled conjugate gradient algorithm [13].

\subsection{Statistical Analysis}

Intra-class correlation coefficient (ICC) was used to measure the concordance between our estimation and the actual AHI. Cohen's kappa $(\kappa)$ assessed the diagnostic ability of the estimated AHI in the four class classification task. Estimated AHI was also evaluated in the three AHI thresholds that define the severity groups to further assess its potentiality as screening tool. Sensitivity (Se), specificity (Sp), and accuracy (Acc) were used for this purpose. Regarding the optimization of the $N_{H}$ and $\alpha$ parameters in the MLP model, a leave-one-out cross-validation (loo-cv) procedure was applied only in the training test. The whole training set, without the loo-cv process, was used to obtain the final MLP model.

\section{Results}

\subsection{MLP Optimization and Training (Training Set)}

Figure 1 shows the optimization of $N_{H}$ and $\alpha$ during the loo-cv procedure. The pair $N_{H}=45$ and $\alpha=0.3$ reached the highest $\kappa(0.597)$. These values were chosen as optimum, and used to train the model with the whole training set.

\subsection{Diagnostic Ability of Our Proposal (Test Set)}

Figure 2 displays a Bland-Altman plot facing the estimated AHI and the actual AHI. A small overestimation of our AHI can be observed $($ mean $=1.2$ ) with the limits of the $95 \%$ confidence interval in the range $(-19.2,21.6)$. Table 2 displays the confusion matrices of both $\mathrm{ODI}_{3}$ alone and the AHI estimated by our MLP model in the test set. $\mathrm{ODI}_{3}$ rightly estimates the severity of $62.0 \%$ of the subjects, showing an $I C C=0.840$ between it and the actual AHI, and a Cohen's $\kappa=0.470$ in the four-class classification task. By contrast, our estimated AHI rightly classifies $75.0 \%$ of subjects into their actual severity degree, achieving ICC = 0.904 and $\kappa=0.614$. Table 3 summarizes the diagnostic statistics for the three AHI thresholds that limit the SAHS-severity levels, i.e., AHI $=5,15$, and $30 \mathrm{e} / \mathrm{h}$. The estimated AHI reaches the highest Acc for the three thresholds, outperforming $\mathrm{ODI}_{3}$ in $\mathrm{AHI}=5$ and $30 \mathrm{e} / \mathrm{h}$. 
Fig. $1 N_{H}$ and $\alpha$ optimization process with loo-cv

Fig. 2 Bland-Altman plot facing estimated and actual AHI
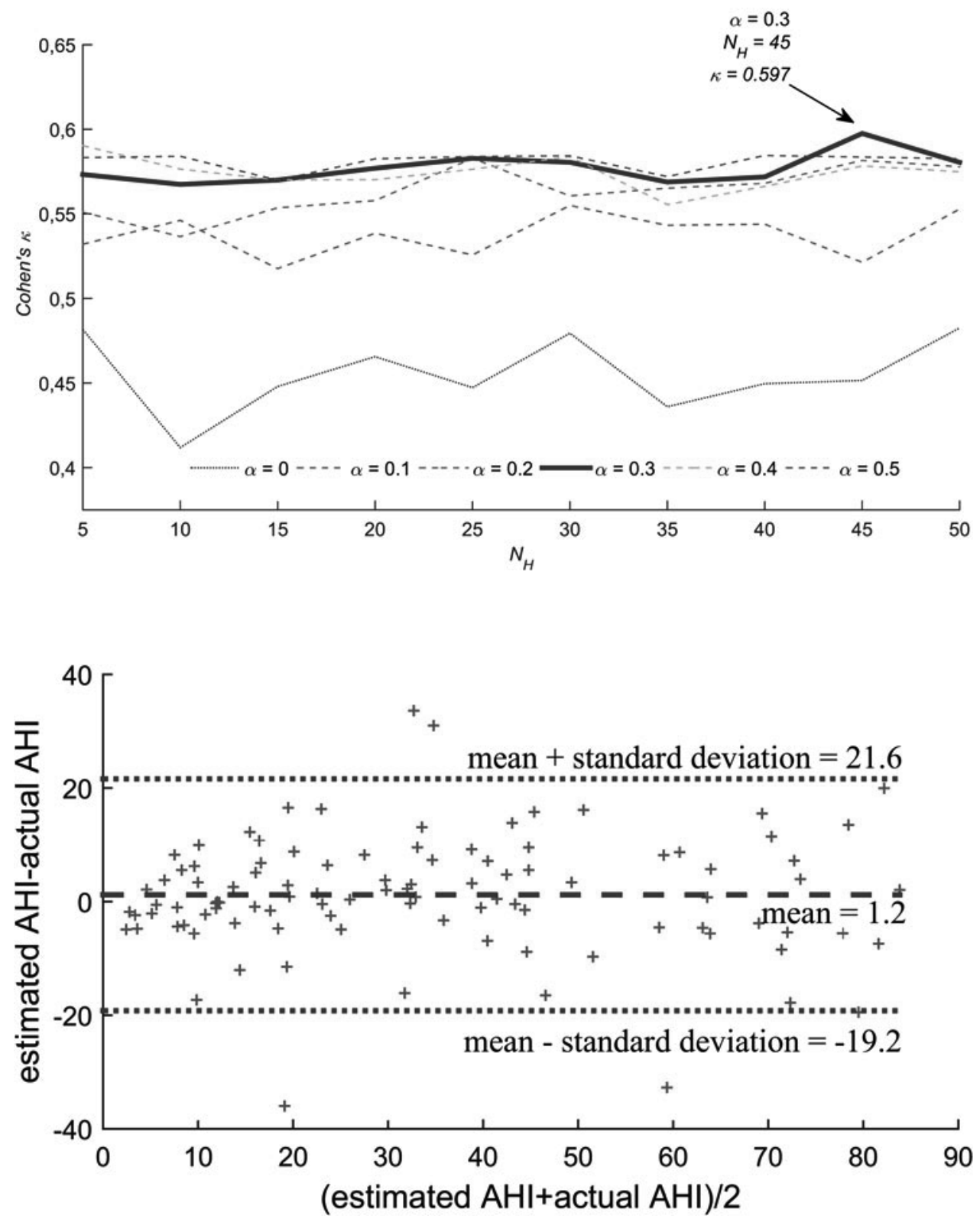

Table 2 Confusion matrices for $\mathrm{ODI}_{3}$ and the AHI estimation derived from our MLP model (Test set). Rows correspond to the actual SAHS severity degree

\begin{tabular}{l|l|l|l|l}
\hline & no-SAHS & Mild & Moderate & Severe \\
\hline $\mathrm{ODI}_{3}$ & \multicolumn{5}{l}{} \\
\hline no-SAHS & $\mathbf{6}$ & 0 & 0 & 0 \\
\hline Mild & 9 & $\mathbf{1 3}$ & 1 & 0 \\
\hline Moderate & 0 & 10 & $\mathbf{1 2}$ & 1 \\
\hline Severe & 1 & 0 & 16 & $\mathbf{3 1}$ \\
\hline Estimated AHI & $\mathbf{3}$ & 3 & 0 & \\
\hline no-SAHS & $\mathbf{3}$ & $\mathbf{1 4}$ & 6 & 0 \\
\hline Mild & 2 & 3 & 12 & 7 \\
\hline Moderate & 1 & 0 & 1 & 46 \\
\hline Severe & 1 & & &
\end{tabular}

Table 3 Evaluation of AHI thresholds (5, 15, and $30 \mathrm{e} / \mathrm{h})$ for both $\mathrm{ODI}_{3}$ and our AHI estimation from MLP (Test set)

\begin{tabular}{|c|c|c|c|}
\hline & $5 \mathrm{e} / \mathrm{h}$ & $15 \mathrm{e} / \mathrm{h}$ & $30 \mathrm{e} / \mathrm{h}$ \\
\hline \multicolumn{4}{|l|}{$\mathrm{ODI}_{3}$} \\
\hline Se. $(\%)$ & 89.4 & 84.5 & 64.6 \\
\hline Sp. (\%) & 100.0 & 96.6 & 98.1 \\
\hline Acc. $(\%)$ & 90.0 & 88.0 & 82.0 \\
\hline
\end{tabular}

Estimated AHI

\begin{tabular}{l|l|l|l}
\hline Se. (\%) & 95.7 & 92.9 & 95.8 \\
\hline Sp. (\%) & 50.0 & 75.9 & 84.6 \\
\hline Acc. (\%) & $\mathbf{9 3 . 0}$ & $\mathbf{8 8 . 0}$ & $\mathbf{9 0 . 0}$ \\
\hline
\end{tabular}




\section{Discussion and Conclusions}

In this study, we obtained an MLP model with ability to estimate AHI from oximetric recordings acquired at patient's home. This model was trained with $\mathrm{ODI}_{3}$ from $\mathrm{SpO}_{2}$ as well as 7 features from PRV that reflect cardiac information not showed by the former. Our proposal reached very high agreement with actual AHI $(I C C=0.904)$ and diagnostic ability $(\kappa=0.614 ; 93.0 \%, 88.0 \%, 90.0 \%$ Acc for 5,15 , and $30 \mathrm{e} / \mathrm{h}$, respectively), outperforming the single use of the widespread clinical parameter $\mathrm{ODI}_{3}$ at each statistics.

Three studies have been found evaluating the diagnostic usefulness of at-home NPO. All of them only analyzed the $\mathrm{SpO}_{2}$ signal. Olson et al. conducted a univariate analysis through delta index which showed moderate $\mathrm{Se} / \mathrm{Sp}$ pairs in the three AHI thresholds $(82.7 \% / 54.2 \%, 88.5 \% / 39.6 \%$, $92.6 \% / 34.1 \%, 5 \mathrm{e} / \mathrm{h}, 15 \mathrm{e} / \mathrm{h}$, and $30 \mathrm{e} / \mathrm{h}$, respectively) [15]. Chung et al. directly assessed the usefulness of at-home $\mathrm{ODI}_{3}$, reaching 87.0, 84.0, 93.7\% Acc for the same AHI thresholds [16]. Finally, Schlotthauer et al. estimated $\mathrm{ODI}_{3}$ by means of the empirical mode decomposition, and its diagnostic assessment for $\mathrm{AHI}=15 \mathrm{e} / \mathrm{h}$ reached $83.8 \% \mathrm{Se}$ and $85.5 \% \mathrm{Sp}$ [17]. Our proposal achieved an overall higher diagnostic ability than those reported in these works.

Notwithstanding these considerations, some limitations need to be pointed out. In accordance with the high prevalence of SAHS, and the pre-test symptoms referred by the patients involved in the study, the number of no-SAHS subjects is low comparing to the other SAHS-severity groups. A higher proportion of them would enhance the soundness of our results. This issue will be addressed in future studies. In addition, although the features extracted from the PRV signal have shown their usefulness, another future goal is the assessment of the information extracted by means of different analytical approaches.

Summarizing, our automatic estimation of the AHI has shown very high diagnostic ability using a MLP model only trained with at-home oximetric recordings. It outperformed the state-of-the-art studies found. Adding PRV features to $\mathrm{ODI}_{3}$ enhanced the performance of the oximetric index alone. These results suggest that the information contained in the PRV signal complement $\mathrm{ODI}_{3}$, leading to an accurate at-home diagnostic alternative.

Acknowledgements This study was partially funded by the projects TEC2014-53196-R and RTC-2015-3446-1 of 'Ministerio de Economía y Competitividad and FEDER', and by VA037U16 of the 'Junta de Castilla y León' and FEDER.

Conflict of Interest The authors declare no conflict of interest.

\section{References}

1. Berry, R. B., et al.: Rules for scoring respiratory events in sleep: update of the 2007 AASM manual for the scoring of sleep and associated events. J. Clin. Sleep. Med., 8, 597, (2012).

2. Lopez-Jimenez, F. et al.: Obstructive sleep apnea: implications for cardiac and vascular disease. Chest, 133, 793-804 (2008).

3. Flemons, W. W., et al.: Home diagnosis of sleep apnea: a systematic review of the literature. Chest, 124, 1543-79 (2003).

4. Penzel, T., et al.: Comparison of detrended fluctuation analysis and spectral analysis for heart rate variability in sleep and sleep apnea. IEEE Trans. Biomed. Eng., 50, 1143-51 (2003).

5. Gutiérrez-Tobal, G. C., et al.: Utility of adaboost to detect sleep apnea-hypopnea syndrome from single-channel airflow. IEEE Trans. Biomed. Eng., 63, 636-46 (2016).

6. Bloch, K. E.: Getting the most out of nocturnal pulse oximetry. Chest, 124, 1628-30, (2003).

7. Sánchez-Morillo, D., et al.: Novel multiclass classification for home-based diagnosis of sleep apnea hypopnea syndrome. Expert Syst. Appl., 41, 1654-62 (2014).

8. Hornero, R., et al.: Nocturnal Oximetry based Evaluation of Habitually Snoring Children. Am. J. Respir. Crit. Care Med., 196, 1591-98 (2017).

9. Gutiérrez-Tobal, et al.: Assessment of time and frequency domain entropies to detect sleep apnoea in heart rate variability recordings from men and women. Entropy, 17, 123-41 (2015).

10. Garde, A., et al.: Development of a screening tool for sleep disordered breathing in children using the phone Oximeter. PloS ONE, 9, e112959 (2014).

11. Lázaro, J., et al.: Pulse rate variability analysis for discrimination of sleep-apnea-related decreases in the amplitude fluctuations of pulse photoplethysmographic signal in children. IEEE J. Biomed. Health. Inform., 18, 240-46, (2014).

12. Marcos, J. V., et al.: Automated prediction of the apnea-hypopnea index from nocturnal oximetry recordings. IEEE Trans. Biomed. Eng., 59, 141-49 (2012).

13. Gutiérrez-Tobal, G. C., et al.: Pattern recognition in airflow recordings to assist in the sleep apnoeahypopnoea syndrome diagnosis. Med. Biol. Eng. Comput., 51, 1367-80 (2013).

14. Magalang, U. J., et al.: Prediction of the apnea-hypopnea index from overnight pulse oximetry. Chest, 124, 1694-1701 (2003).

15. Olson, L. G., et al.: Prediction of sleep-disordered breathing by unattended overnight oximetry. J. Sleep. Res., 8, 51-55 (1999).

16. Chung, F., et al.: Oxygen desaturation index from nocturnal oximetry: a sensitive and specific tool to detect sleep-disordered breathing in surgical patients. Anesth. Analg., 114, 993-1000 (2012).

17. Schlotthauer, et al.: Screening of obstructive sleep apnea with empirical mode decomposition of pulse oximetry. Med. Eng. Phys., 36, 1074-80 (2014). 